\title{
The clinicopathological features of three babies with osteogenesis imperfecta resulting from the substitution of glycine by valine in the pro $\alpha 1$ (I) chain of type I procollagen
}

\author{
W G Cole, E Patterson, J Bonadio, P E Campbell, D W Fortune
}

\section{Department of Paediatrics, University of Melbourne, Royal Children's Hospital, Flemington Road, Parkville, Victoria 3052, Australia. W G Cole \\ Department of Anatomical Pathology, Royal Children's Hospital, Melbourne, Australia. P E Campbell}

Department of Anatomical Pathology, Royal Women's Hospital, Melbourne, Australia. D W Fortune

Howard Hughes Medical Institute and the Department of Pathology, The Michigan, Ann Arbor Michigan 48109-0650,

USA.

E Patterson

$\mathrm{J}$ Bonadio

Correspondence to Professor Cole.

Received 26 June 1991 Accepted 31 July 1991.

\begin{abstract}
The features of three babies with perinatal lethal osteogenesis imperfecta (OI II) resulting from substitutions of glycine by valine in the triple helical domain of the $\alpha 1$ (I) chain of type I collagen were studied. The babies were heterozygous for this substitution at residue 1006 in case 1 (OI35), 973 in case 2 (OI59), and 256 in case 3 (OI7B). OI35 had the most severe clinical form, OI IIC, with premature rupture of membranes, severe antepartum haemorrhage, stillbirth, severe short limbed dwarfism, and extreme osteoporosis. OI59 was a better formed baby but was also born prematurely as a result of premature rupture of membranes and severe antepartum haemorrhage. She had the radiographic features of OI IIA. OI7B was born at term and also had the radiographic features of OI IIA. Pathological examination of the skeletons of OI35 and OI59 showed grossly deficient intramembranous and endochondral ossification. Trabecular bone was sparse in the long bones and vertebrae. The trabeculae contained a cartilage core and an overlying layer of woven bone or osteoid. The diaphyses lacked cortical bone. The periosteal fibroblasts of 0135 contained grossly distended rough endoplasmic reticulum consistent with the $53 \%$ reduction in collagen secretion by cultured dermal fibroblasts. The aorta, skin, and lungs were hypoplastic in OI35 and OI59. The findings in this study show that glycine substitutions by valine in Gly-X-Y triplets, from glycine 256 to glycine 1006, of the triple helical domain of $\alpha 1$ (I) chains produce the OI II phenotype. The phenotype was most severe in the baby with the most carboxy-terminal substitution.
\end{abstract}

Osteogenesis imperfecta (OI) is a heterogeneous genetic disorder that produces bone fragility, abnormal blueness of the sclerae, dentinogenesis imperfecta, and premature deafness. ${ }^{1}$ There are four main clinical types of OI and the perinatal lethal type, type II OI or OI II, is the focus of this report. ${ }^{23}$

OI II usually results from heterozygous mutations of the COL1A1 gene that encodes the pro $\alpha 1(\mathrm{I})$ chains and less frequently results from mutations of the COL1A2 gene that encodes the pro $\alpha 2(\mathrm{I})$ chain of type I procollagen. ${ }^{4}$ Mutations of the COL1A1 gene alter the structure of the main triple helical domain or, less frequently, the carboxy-propeptide extension of the pro $\alpha 1(I)$ chain. In babies with the latter type of defect, the abnormal pro $\alpha \mathrm{l}(\mathrm{I})$ chains associate with other chains to form mutant type I procollagen molecules that are rapidly degraded. ${ }^{5}$ As a result, only a small amount of normal type I procollagen is secreted from cultured fibroblasts and the tissues contain correspondingly small amounts of normal type I collagen. In these babies, the OI II phenotype appears to be the result of a severe deficiency of normal type I collagen in the tissues. $^{6}$

Cultured fibroblasts from babies with helical mutations produce normal type I procollagen and abnormal type I procollagen molecules containing one or two mutant chains. ${ }^{78}$ The mutant molecules are secreted less efficiently than the normal ones and the level of intracellular collagen degradation is increased. In these babies, the OI II phenotype appears to result from a deficiency of normal type I collagen and the presence of mutant type collagen in the tissues. ${ }^{9}$

Most of the structural mutations of the triple helical domain of the $\alpha 1$ (I) chain involve the substitution of a glycine residue in one of the 338 Gly-X-Y repeating triplets. ${ }^{4}$ These substitutions destabilise and delay the formation of the triple helix, and allow excessive post-translational hydroxylation and glycosylation of lysine residues on the amino-terminal side of the mutation. ${ }^{70-12}$ As a result, correlations have been sought between the type and site of glycine substitutions and the resulting clinical phenotypes. The position of glycine substitutions by cysteine appears to determine the clinical phenotype while such correlations are less evident with other types of glycine substitutions. ${ }^{913}$ In this paper, we explore these relationships further in three babies with OI II resulting from heterozygous substitutions of glycine by valine at positions $256,{ }^{14}$ $973,{ }^{15}$ and $1006^{15}$ of the triple helical domain of the $\alpha 1(I)$ chain.

\section{Case reports}

The main details of the parents, pregnancies, and babies are summarised in the table. 
Features of the parents, pregnancies, and babies.

\begin{tabular}{llll}
\hline Features & OI35 (Gly 1006) & OI59 (Gly 973) & OI7B (Gly 256) \\
\hline Father's age & 32 & 37 & 29 \\
Mother's age & 25 & 33 & 27 \\
Parity & 1 & 0 & 2 \\
Gravida & 28 & 2 & 3 \\
Gestation (w) & Large antepartum & 30 & 40 \\
Pregnancy & haemorrhage & Large antepartum & Normal \\
Delivery & Vaginal & Caesarian section & Vaginal \\
Sex & Female & Female & Female \\
Head circumference $(\mathrm{cm})$ & & $24^{*}$ & \\
Crown-heel length $(\mathrm{cm})$ & $26^{*}$ & $30^{*}$ & \\
Birth weight $(\mathrm{g})$ & $650^{*}$ & $1000^{*}$ & \\
Survival (days) & Stillborn & IIA & 120 \\
Type of OI $\dagger$ & IIC & IIA
\end{tabular}

* Value less than the 10th centile for an Australian hospital population. ${ }^{16}$

+ Sillence classification. ${ }^{12}$

CASE 1 (OI35)

This girl was the second child of healthy, unrelated parents. There was a threatened miscarriage at 12 weeks of gestation and no fetal movements were noted during the pregnancy. Ultrasound examination at 26 weeks of gestation showed that the skull was poorly echogenic and the heart, which was contracting normally, occupied a greater than normal proportion of the chest. The biparietal skull diameter was $7 \cdot 14 \mathrm{~cm}$ and the occipitofrontal diameter was $7 \cdot 89 \mathrm{~cm}$. At 28 weeks of gestation, spontaneous rupture of the membranes occurred and was immediately followed by severe vaginal bleeding and a tense tender uterus. There were no fetal heart sounds and an abdominal radiograph showed an abnormal fetus. The baby was stillborn after a vaginal delivery.

The baby girl was a short limbed dwarf (fig 1). The calvarium was large and soft, the

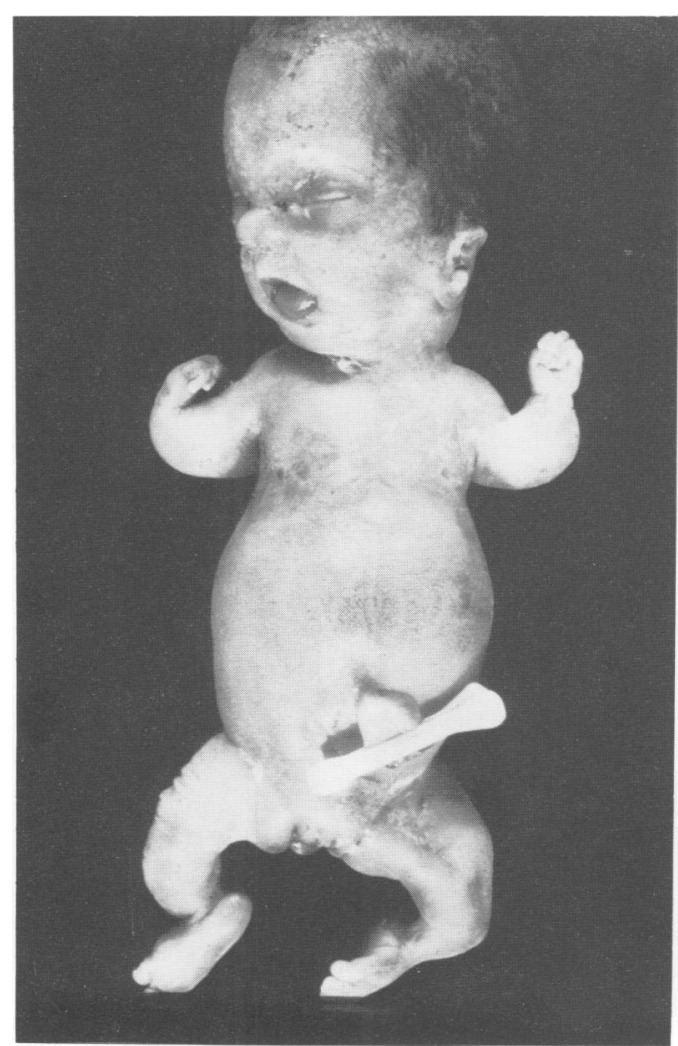

Figure 1 OI35: clinical appearance. midface was hypoplastic, and the lower jaw was small. In keeping with the ultrasound findings, the upper chest was narrow and the abdomen protuberant. The limbs were short and bowed. There was widespread crepitus of the ribs and limb bones.

The radiographs showed severe generalised osteopenia, a malformed skeleton, and multiple fractures (fig 2). The calvarium was not ossified and only a minimal amount of bone was present in the base of the skull and jaws. The mid thoracic vertebral bodies were flat whereas the other vertebrae were of normal shape. The proximal ribs were short, thin, and wavy and contained numerous fractures. The lower ribs were also short but they were broader and continuously beaded. The long

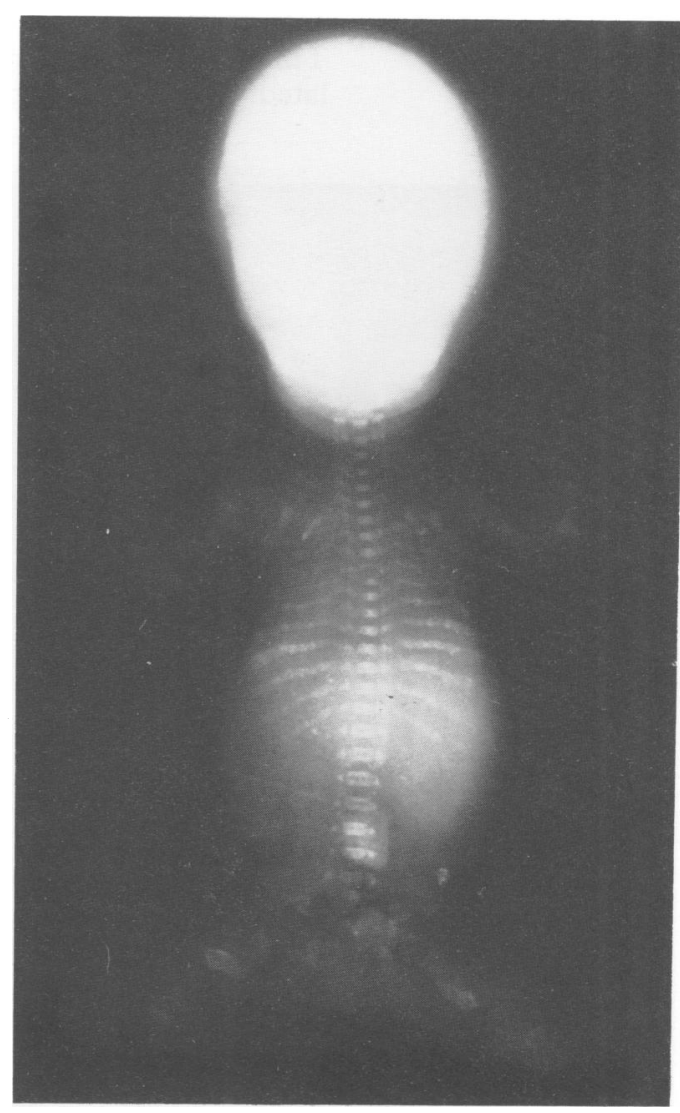

Figure 2 OI35: anteroposterior radiograph. The skull is poorly ossified, the ribs are short and continuously beaded, several thoracic vertebrae are flat, and the long bones are broad and crumpled. 


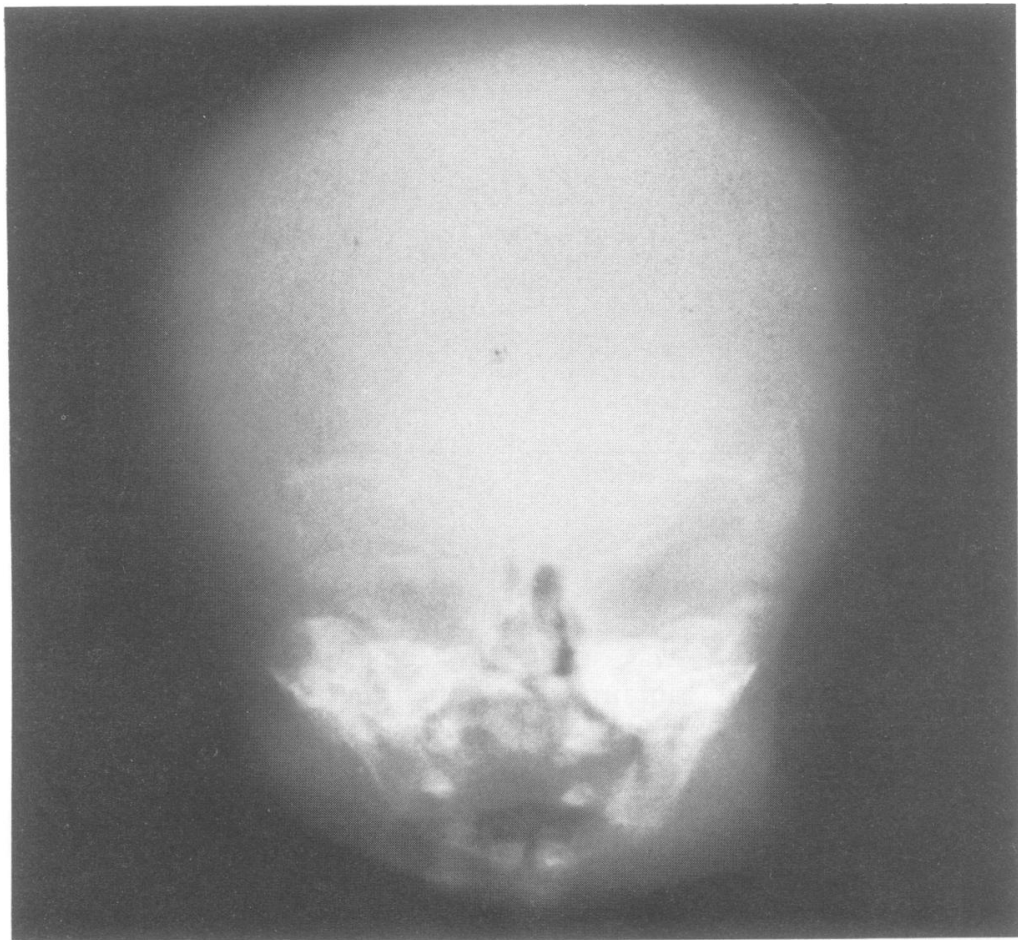

Figure 3 OI59: anteroposterior skull radiograph showing minimal ossification.

bones were short, broad, and crumpled. The diaphyses were as wide as the metaphyses and there was no cortical bone. The radiographic features were consistent with those ascribed to type IIC OI. ${ }^{23}$

CASE 2 (O159)

This girl was the first child of healthy, unrelated parents. The previous pregnancy had

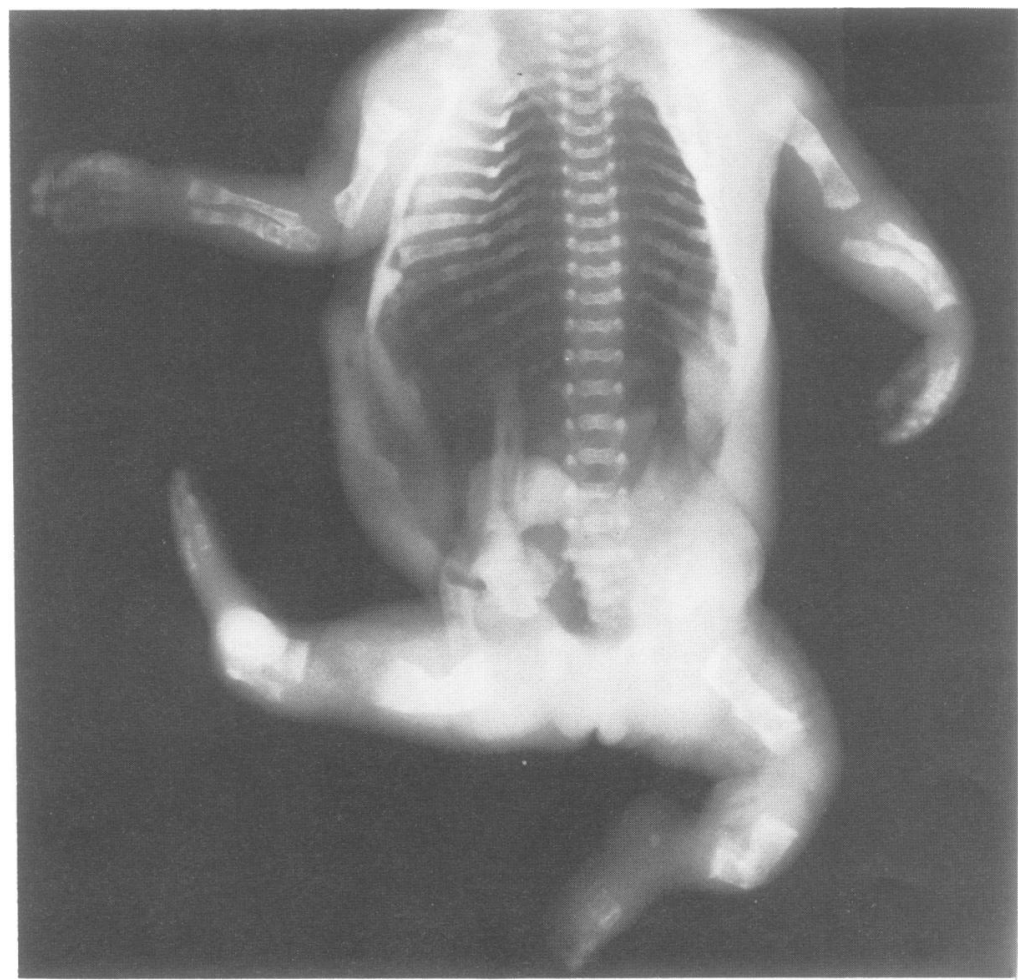

Figure 4 OI59: anteroposterior radiograph at necropsy showing beaded ribs, generalised platyspondyly, and short, broad, and crumpled long bones.

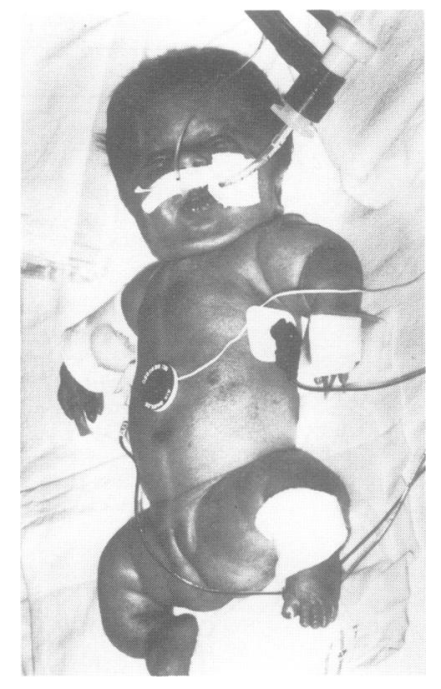

Figure 5 OI7B: clinical appearance.

terminated spontaneously at 6 weeks of gestation. A set of twins and another baby from a previous marriage of the father had died from prematurity and bronchopulmonary dysplasia. None of the dead babies showed signs of OI or other connective tissue disorders.

The pregnancy of OI59 was apparently normal until 30 weeks of gestation when the membranes ruptured with a severe antepartum haemorrhage for which an emergency caesarian section was undertaken. The female baby died 20 minutes after delivery.

The baby weighed $1000 \mathrm{~g}$ and had the typical features of OI II. She had a large, globular, and soft skull and short bowed limbs containing multiple fractures. The chest and abdominal circumferences were 18 and $17 \mathrm{~cm}$, respectively.

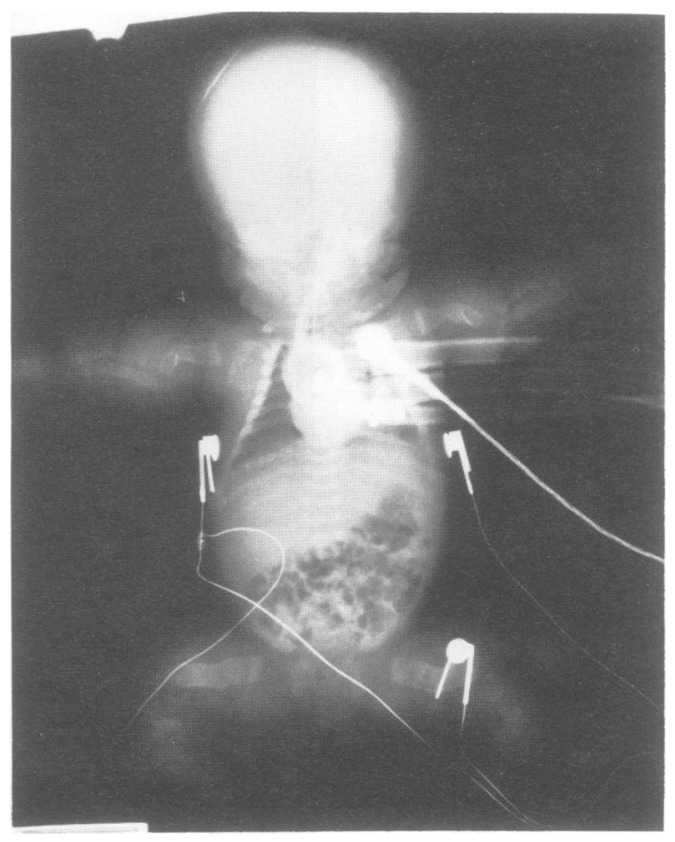

Figure 6 OI7B: anteroposterior radiograph showing poor ossification of the skull, continuously beaded ribs, and generalised platyspondyly. The long bones are short, broad, and crumpled. 


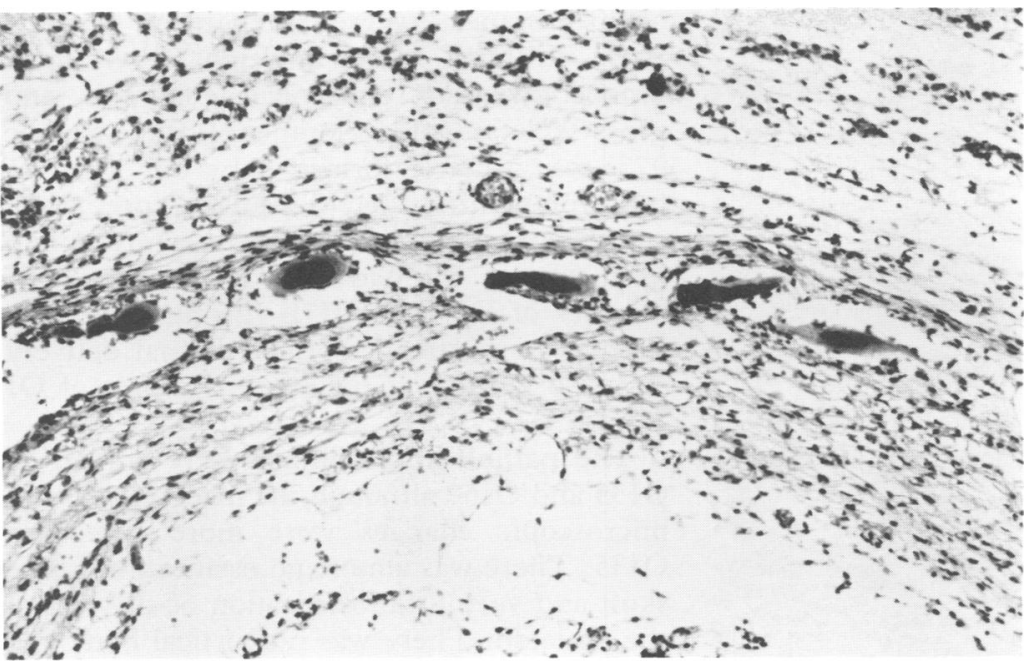

Figure 7 Calvarium of OI35. The bone is represented by tiny plates of calcifying osteoid in a loose collagenous matrix. There is no separation of inner and outer tables.

The radiographs showed severe generalised osteopenia and multiple fractures. The calvarium showed minimal calcification, the orbits were poorly defined, but there was some calcification of the petrous temporal bones (fig 3). The maxilla and mandible were hypoplastic and poorly ossified. There was generalised severe platyspondyly which was worse in the thoracic than the lumbar spine (fig 4). The ribs were irregular and discontinuously beaded. The long bones were short, crumpled, and lacked cortical bone. The femora and tibiae did not show any modelling as the diaphyses were as wide as the metaphyses. The long bones of the upper limbs had a similar appearance although there was a small amount of modelling of the diaphyses. The radiographical features were consistent with those ascribed to type IIA OI. ${ }^{23}$

CASE 3 (OI7B)

This girl was the third child of healthy, unrelated parents. The previous daughters were

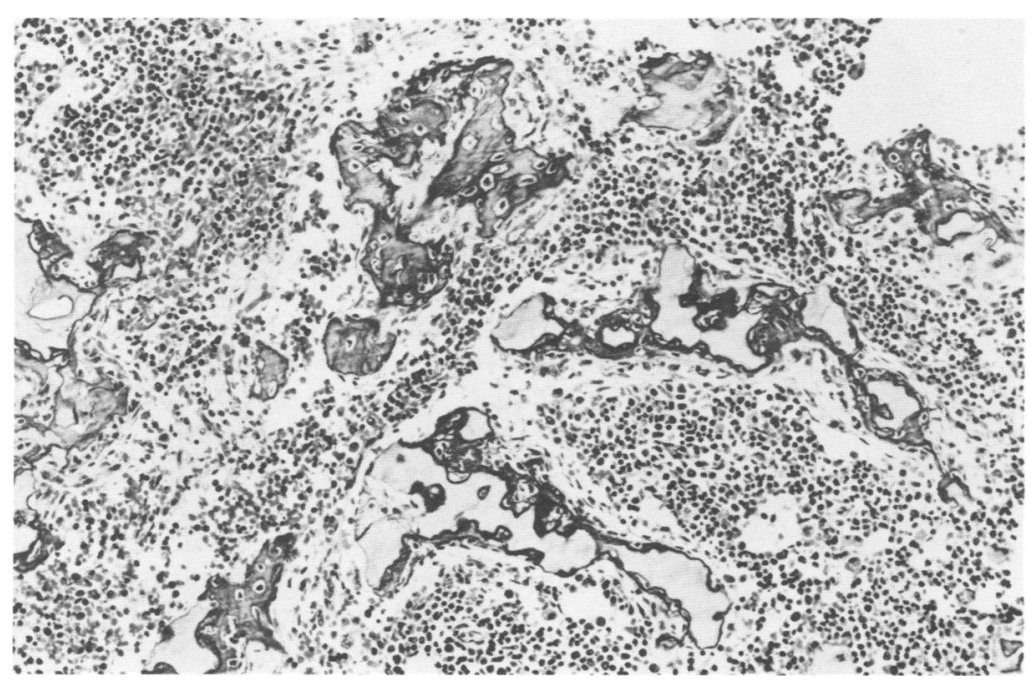

Figure 8 Vertebral body of OI59 showing an abundance of bone marrow and few trabeculae. Most of the trabeculae consist of cartilage cores with a narrow rim of osteoid. normal. The female proband was born at term after an uncomplicated pregnancy. She had a large, soft calvarium, blue sclerae, small chest, and short bowed limbs (fig 5). The radiographs showed minimal calcification of the calvarium, base of the skull, and facial bones (fig 6). There was generalised platyspondyly. The chest was narrow and the ribs were broad and continuously beaded. The long bones were short, broad, and crumpled. The radiographical features were of type IIA OI. ${ }^{23}$

The proband experienced respiratory difficulty at birth and was placed on a ventilator which was continued until her death at 4 months of age.

\section{PATHOLOGICAL STUDIES}

Necropsies of OI35 and OI59 were undertaken and the findings were very similar. In each baby, the skull was globular and extremely soft. Most of the calvarium consisted of an unossified membrane and even the petrous temporal bones were soft. The chest was narrow and the ribs showed healing fractures along their diaphyses and costochondral junctions. The long bones were short and bowed and contained multiple fractures and irregular calluses.

The viscera were macroscopically normal, except for the lungs which were hypoplastic, and the aorta was thin. In OI35, there was also leucomalacia of the periventricular regions of the brain. The skin was soft and thin in both babies.

Histological examination of the calvarium showed minimal intramembranous bone formation. There were a few islands of calcifying osteoid (fig 7) but most of the calvarium consisted of a membrane of fused galea aponeurotica and dura mater. Histological examination of costochondral junctions and growth plates of the femora showed that the cartilaginous parts of the growth plates were normal. The cancellous bone of the metaphyses of long bones and of the vertebral bodies showed sparse trabeculae which contained cores of calcified cartilage covered by woven bone or by a thin layer of osteoid (fig 8). The diaphyses of the ribs and femora also contained sparse amounts of trabecular bone (fig 9). There was no evidence, using polarising light microscopy, of any lamellar bone or haversian systems. The osteoblasts, which were abundant along the surface of the trabeculae and within areas of woven bone, were surrounded by little matrix. They were plump and basophilic but did not appear to mature into osteocytes. The periosteum of the rib and femoral diaphysis was almost as thick as the underlying pseudocortex of trabecular woven bone (fig 9 ).

In OI35, electron microscopy of the periosteum of the femur showed fibroblasts with grossly distended rough endoplasmic reticulum (fig 10). The periosteum also contained osteoblastic cells which contained abundant amounts of rough endoplasmic reticulum. However, the rough endoplasmic reticulum of these cells was not distended (fig 11). 


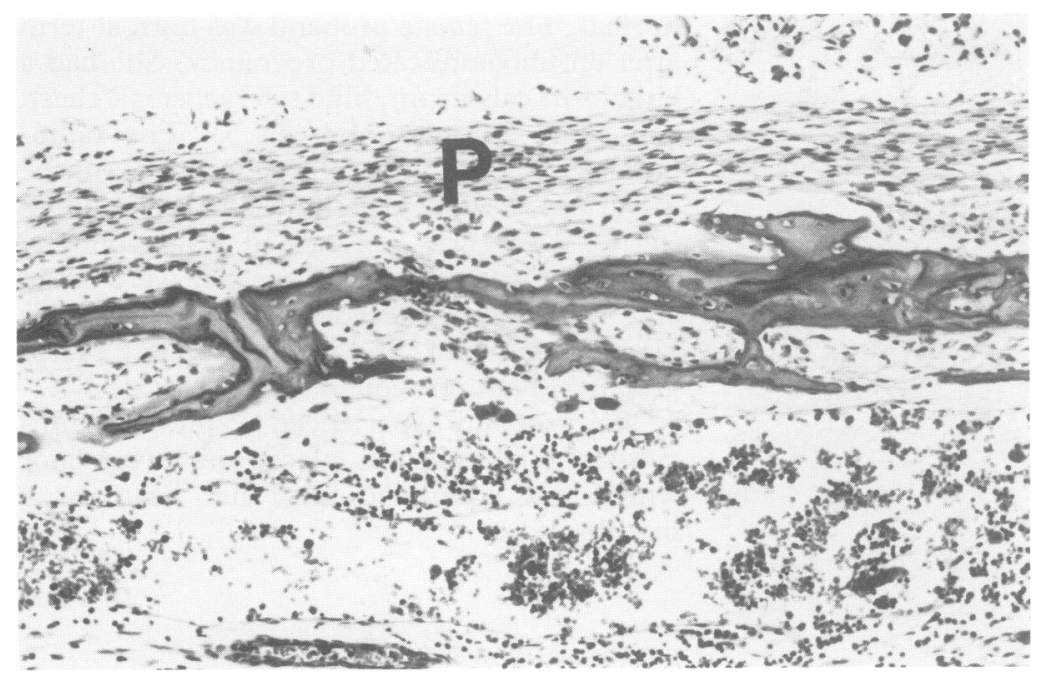

Figure 9 Femoral diaphysis of OI35 showing the periosteum $(P)$ overlying the thin cortex which contains poorly formed lamellae of woven bone.

Histological examination of the viscera also showed that the collagenous supporting structures were thin and delicate. The tunica media and the adventitial layer of the aorta were thinner than normal (fig 12). The sclera of OI59 had an average thickness of $200 \mu$ and was composed of thin delicate collagen fibres.

\section{Discussion}

The three babies in this study had OI II because of glycine substitutions by valine within the carboxy-terminal $75 \%$ of the triple helical domain of the $\alpha 1(I)$ chain of type I collagen. The glycine substitutions occurred in Gly-X-Y triplets in which glycine in the first position is essential for the normal formation of the triple helix. ${ }^{7}$

OI35 (glycine 1006) had the most severe clinical form, OI IIC, with premature rupture

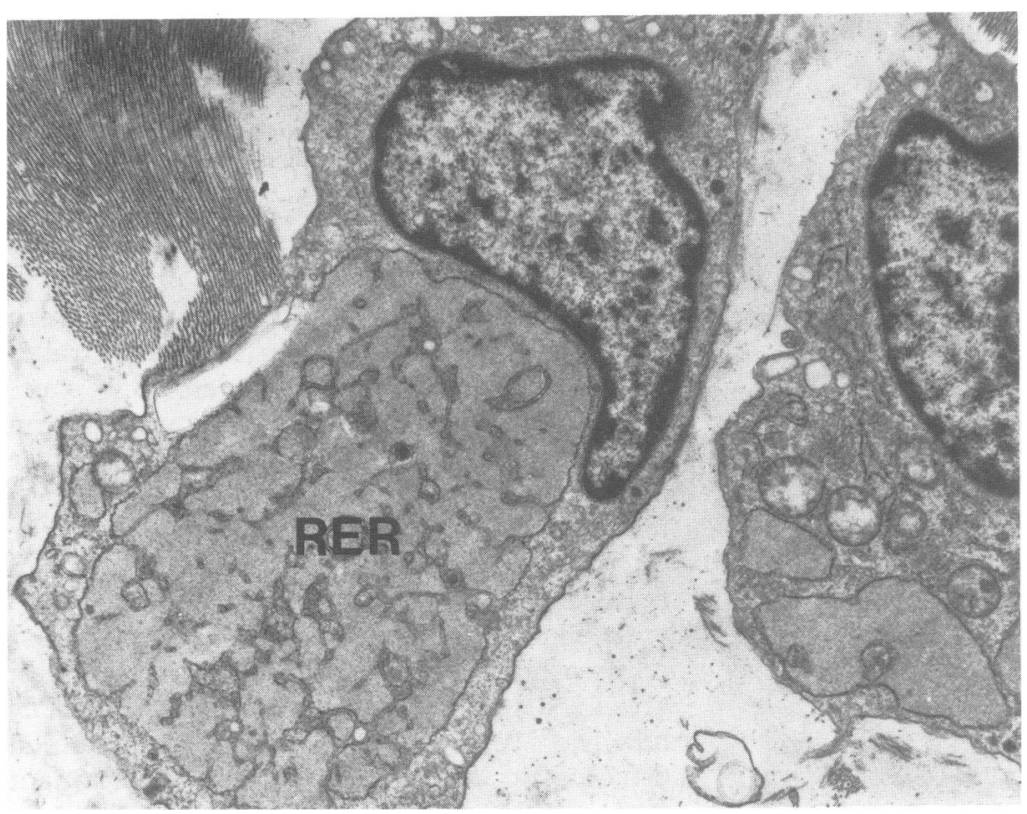

Figure 10 Electron micrograph of periosteal fibroblasts of OI35. All the fibroblasts in the femoral periosteum contained grossly dilated rough endoplasmic reticulum (RER). of membranes, severe antepartum haemorrhage, stillbirth, low birth weight, severe short limbed dwarfism, extreme osteoporosis, and gross skeletal maldevelopment. OI59 (glycine 973) was a better formed baby but was also born prematurely as a result of premature rupture of membranes and severe antepartum haemorrhage. She had the typical radiographic features of OI IIA. OI7B (glycine 256) was born at term after a normal vaginal delivery and also had the radiographic features of OI IIA.

The pathological findings were similar in OI35 and OI59 although the macroscopic and microscopic changes were more severe in OI35. There was almost no ossification in the skull and very little ossification of the rest of the skeleton. There was no cortical bone and no evidence of organised collagen matrices as seen in normal lamellar bone and haversian systems. The macroscopic and microscopic findings suggested that the osteoblasts were producing a minimal amount of extracellular matrix that was abnormal in composition. The osteoblasts did not mature into osteocytes and normal bone remodelling was absent.

The abnormal collagen metabolism previously reported in these babies provides some explanations for the clinicopathological findings. Cultured dermal fibroblasts from each baby produced a mixture of normal and mutant type I collagen molecules. ${ }^{7814}$ Chains from the abnormal molecules migrated slowly on electrophoresis owing to the overmodification of lysine residues on the amino-terminal side of the glycine substitution. In each case, the distribution of the overmodified lysine residues correlated with the site of the glycine substitution. The secretion of the mutant molecules was retarded. In OI35, secretion was reduced to $47 \%$ of normal while in OI59 it was $73 \%$ of normal. The gross reduction in secretion by OI35 fibroblasts provided an explanation for the gross dilatation of the rough endoplasmic reticulum observed in periosteal fibroblasts. In contrast, periosteal osteoblasts from OI35 did not contain distended rough endoplasmic reticulum. The reason for the different appearances of the rough endoplasmic reticulum in these cell types was not determined. The osteoblastic cells may have been in a proliferative rather than matrix synthesising phase of growth or alternatively osteoblasts may be able to secrete the mutant collagen more efficiently than fibroblasts.

Analysis of tissue collagens in OI35 and OI59 confirmed that both normal and mutant type I collagen was present in the extracellular matrix in vivo. ${ }^{8}$ In OI35 dermis, the concentration of type I collagen was $24 \%$ of normal while in OI59 it was $28 \%$ of normal. In both samples, there was a reduced amount of acetic acid soluble collagen, which suggested that mutant collagen was degraded if it was not rapidly incorporated into the more insoluble matrix. In keeping with the dermal results, OI35 bone contained $59 \%$ of the normal amount of collagen while OI59 bone contained $73 \%$ of the normal amount. Although these results, which were obtained by determining 


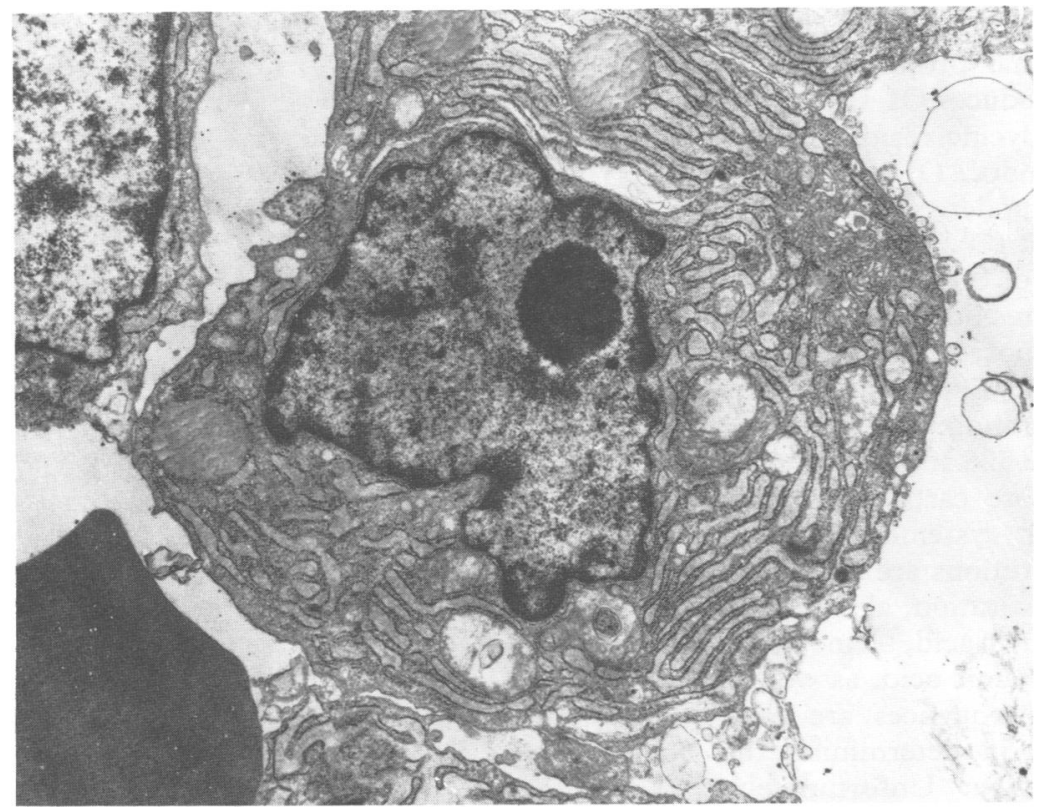

Figure 11 Electron micrograph of periosteal osteoblast of OI35. Osteoblasts in the deep layer of the femoral periosteum were normal in appearance. The rough endoplasmic reticulum was not distended.
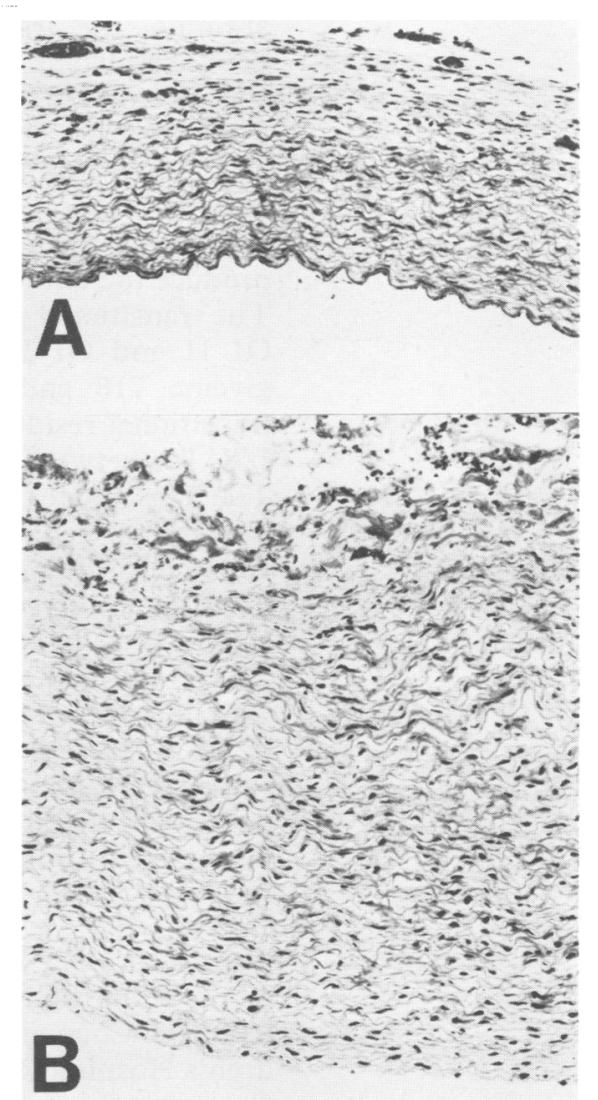

the concentration of collagen per $\mathrm{mg}$ dry weight of decalcified bone matrix, showed a reduced amount of bone collagen, the radiographic and pathological findings indicated that the amount of bone matrix was reduced to a much greater extent. The disparity in these results reflects the way in which bone mass was determined in the tissue study. A better biochemical estimate of bone mass would have been obtained if the total collagen and calcium contents of control and OI long bones had been determined.

The pathological findings in the present study were similar to those observed in a transgenic mouse model of OI II in which glycine 859 of the $\alpha 1$ (I) chain was substituted by cysteine. ${ }^{17}$ The tissues were grossly disorganised and contained a markedly reduced amount of total collagen even when the mutant transgene was expressed at low levels. These findings confirm the devastating effect of even small amounts of the mutant collagen in the tissues.

Although all of the babies in the present study died, the phenotype produced by the glycine 1006 substitution by valine was more severe than the phenotypes produced by the substitutions at glycine 973 and glycine 256 . There was a gradient in the period of gestation from 28 weeks in OI35 (glycine 1006) to 30 weeks in OI59 (glycine 973) and 40 weeks in OI7B (glycine 256). The premature rupture of membranes and antepartum haemorrhage in OI35 and OI59 may have been the result of abnormal extracellular matrix of the membranes although this possibility was not studied further. There was also a similar gradient in the survival of the babies although the longer survival of OI7B may have been because of prolonged artificial ventilation. The radiographic and pathological findings also indicated that OI35 was the most severely
Figure 12 The aorta from ( $A$ ) OI59 and (B) control. The tunica media of OIS9 was thinner $(200 \mu)$ than the tunica media of age and site matched control aorta $(600 \mu)$. The adventitia, shown at the top of each panel, was also thin with loosely arranged collagen in OIS9.

affected baby. However, some of the differences noted between the babies may reflect differences in their genetic backgrounds.

The results of the present study are similar to those observed previously in babies with glycine substitutions by arginine at positions 391,667 , and 976 of the $\alpha 1(\mathrm{I})$ chain of type I collagen. ${ }^{9}$ These babies had OI II and were subclassified as IIB (glycine 976), IIA/IIB (glycine 667), and IIA (glycine 391). None of them resembled OI35 (glycine 1006) with OI IIC. The glycine 976 substitution by arginine produced OI IIB whereas the nearby glycine 973 substitution by valine produced OI IIA. However, the substitution of glycine 391 by arginine and glycine 256 by valine produced the OI IIA phenotype. Substitutions of glycine $847^{18}$ and $550^{19}$ by arginine were also reported to produce OI II, while the substitution of glycine $154^{13}$ by arginine produced OI III.

The genotype-phenotype relationships observed for substitutions of glycine by arginine or valine in the first position of Gly-X-Y triplets in the $\alpha \mathrm{l}(\mathrm{I})$ chain differ from those reported for glycine substitutions by cysteine. OI II phenotypes were observed with substitutions by valine as far amino-terminal as glycine $256 .{ }^{14}$ However, the transitional glycine residue between the sites producing the OI II and milder phenotypes is unknown as the only reported cases of glycine substitutions by valine in the $\alpha 1(\mathrm{I})$ chain are the three cases described here. For glycine substitutions by 
arginine, the transitional glycine residue is between glycine $154^{13}$ that produces OI III and glycine $391^{9}$ that produces OI II. Substitutions by cysteine at glycine residues $988,{ }^{20}$ $904,,^{21} 748,22$ and $718^{13}$ produce OI II, substitutions at $526^{13}$ produce moderately severe OI III, while substitutions at $178,{ }^{23} 175,{ }^{24}$ and $94^{13}$ produce the milder OI I or OI IV phenotypes. The transitional glycine residue separating the OI II and OI III phenotypes lies between glycine 718 and glycine 526, whereas the transitional residue separating OI III and OI I/IV lies between glycine 526 and glycine 178 . The reasons for the more carboxy-terminal transitional regions with cysteine than with valine or arginine substitutions are unknown The type of glycine substitution, also including substitutions by aspartic acid, serine, alanine, tryptophan, and glutamic acid, as well as the site and surrounding sequences, are likely to be important factors in determining the severity of the phenotype. Unfortunately, patients have not yet been identified with different substitutions of the same glycine residue.

The work by WGC was undertaken with grants from the National Health and Medical Research Council of Australia, the Royal Children's Hospital Research Foundation, and the Osteogenesis Imperfecta Foundation. The work by JB was supported by National Institutes of Health Grant AR38473. JB is an Assistant Investigator of the Howard Hughes Medical Institute. The authors wish to thank Mr M Ashdown for the electron microscopy.

1 Sillence DO, Senn AS, Danks DM. Genetic heterogeneity in osteogenesis imperfecta. $f$ Med Genet 1979;16:101-16. 2 Sillence DO, Barlow KK, Garber AP, Hall JG, Rimoi DL. Osteogenesis imperfecta type II. Delineation of the phenotype with reference to genetic heterogeneity. $A m \mathcal{F}$ Med Genet 1984;17:407-23.

3 Thompson EM, Young ID, Hall CM, Pembrey ME. Recurrence risks and prognosis in severe sporadic osteogenesis imperfecta. F Med Genet 1986;24:390-405.

4 Cole WG. Osteogenesis imperfecta. Baillieres Clin Endocrinol Metab 1988;2:242-65.

5 Bateman JF, Lamande SR, Dahl HHM, Chan D, Mascara $T$, Cole WG. A frameshift mutation results in a truncated non-functional carboxy-terminal propeptide of type procollagen in costeogenesis imperfecta $\not \mathcal{B i o l}$ Chem 1989;264:10960-4.

6 Cole WG, Campbell PE, Rogers JG, Bateman JF. The clinical features of osteogenesis imperfecta resulting from a non-functional carboxy terminal proal(I) propeptide of a non-functional carboxy terminal proal(I) propeptide of type I procollagen and a severe deficiency of normal
collagen in tissues. $\mathcal{f}$ Med Genet 1990;27:545-51.

7 Bateman JF, Mascara T, Chan D, Cole WG. Abnormal type I collagen metabolism by cultured fibroblasts in lethal perinatal osteogenesis imperfecta. Biochem $f$ 1984;217:103-15.

8 Bateman JF, Chan D, Mascara T, Rogers JG, Cole WG. Collagen defects in lethal perinatal osteogenesis imperfecta. Biochem f 1986;240:699-708.

9 Cole WG, Chow CW, Rogers JG, Bateman JF. The clinical features of three babies with osteogenesis imperfecta resulting from the substitution of glycine by arginine in the pro $\alpha 1(\mathrm{I})$ chain of type I procollagen. $\mathcal{F}$ Med Genet 1990;27:228-35.

10 Bonadio JF, Holbrook KA, Gelinas RE, Jacob J, Byers PH. Altered triple helical structure of type-I procollagen in lethal perinatal osteogenesis imperfecta. $\mathcal{f}$ Biol Chem 1985;260:1734-42.

11 Bonadio JF, Byers PH. Subtle structural alterations in the chains of type-I procollagen produce osteogenesis imperfecta type-II. Nature 1985;316:363-6.

12 Steinmann B, Rao VH, Vogel A, Bruckner P, Gitzelmann $\mathrm{R}$, Byers PH. Cysteine in the triple-helical domain of one allelic product of the alpha-1 (I) gene of type-I collagen produces a lethal form of osteogenesis imperfecta. $₹$ Biol Chem 1986;259:11129-38.

13 Starman BJ, Eyre D, Charbonneau H, et al. Osteogenesis imperfecta: the position of substitution of glycine by cysteine in the triple helical domain of the proal(I) chains of type I collagen determines the clinical phenotype. $\mathcal{f}$ Clin Invest 1989;84:1206-14.

14 Patterson E, Smiley E, Bonadio J. RNA sequence analysis of a perinatal lethal osteogenesis imperfecta mutation. $\mathfrak{f}$ Biol Chem 1989;264:10083-7.

15 Lamande SR, Dahl HHM, Cole WG, Bateman JF. Characterization of point mutations in the collagen COL1Al and COL1A2 genes causing lethal perinatal osteogenesis imperfecta. I Biol Chem 1989;264:15809-12.

16 Kitchen WH, Robinson HP, Dickinson AJ. Revised intrauterine growth curves for an Australian hospital population. Aust Paediatr f 1983;19:157-61.

17 Stacey A, Bateman JF, Choi T, Mascara T, Cole WG, Jaenisch R. Mutagenesis of the mouse proal(I) collagen gene: substitution of a glycine results in osteogenesis mperfecta in cultured cells and transgenic mice. Nature 1988;332:131-6.

18 Wallis GA, Starman BJ, Schwartz MF, Byers PH. Substitution of arginine for glycine at position 847 in the triplehelical domain of type I collagen produces lethal osteogenesis imperfecta. $\mathcal{F}$ Biol Chem 1990;265:18628-33.

19 Wallis GA, Starman BJ, Zinn AB, Byers PH. Variable expression of osteogenesis imperfecta in a nuclear family is explained by somatic mosaicism for a lethal point mutation in the $\alpha 1$ (I) gene (COL1A1) of type I collagen in mutation in the $\alpha 1$ (I) gene (COL1A1) of type

20 Cohn DH, Byers PH, Steinmann B, Gelinas RE. Lethal osteogenesis imperfecta resulting from a single nucleotide osteogenesis imperfecta resulting from a single nucleotide
change in one human alpha 1 (I) collagen allele. Proc Natl change in one human alpha 1 (I)
Acad $S$ ci USA 1986;83:6045-7.

21 Constantinou CD, Nielsen KB, Prockop DJ. A lethal variant of osteogenesis imperfecta has a single base mutation that substitutes cysteine for glycine 904 of the alpha 1 (I) chain of type I procollagen. The asymptomatic mothe has an unidentified mutation producing an overmodified and unstable type I procollagen. $\mathcal{f}$ Clin Invest 1989 83:574-84.

22 Vogel BE, Doely R, Kadler KE, Hojima Y, Engel J, Prockop DJ. A substitution of cysteine for glycine 748 of the alpha 1 chain produces a kink at this site in the procollagen I molecule and an altered $\mathrm{N}$-proteinase cleavage site over $225 \mathrm{~nm}$ away. F Biol Chem 1988; 263:19249-55.

23 Valli $M$, Mottes $M$, Tenni $R$, et al. A de novo $G$ to $T$ transversion in a pro- $\alpha 1(\mathrm{I})$ collagen gene for a moderate case of osteogenesis imperfecta. Substitution of cysteine for glycine 178 in the triple helical domain. $f$ Biol Chem for glycine 178 in

24 de Vries WN, De Wet WJ. The molecular defect in an autosomal dominant form of osteogenesis imperfecta. autosis of type I procollagen containing cysteine in the triple-helical domain of pro-alpha $1(\mathrm{I})$ chains. $f \mathrm{Biol}$ Chem 1986;261:9056-64. 